\title{
EFECTOS DEL ENTRENAMIENTO ESTRUCTURADO EN ESCOLARES DEL NORTE ANTIOQUEÑO
}

EFFECTS OF STRUCTURED TRAINING IN ANTIOQUIAN SCHOOL STUDENTS

Carlos Alberto Agudelo Velásquez ${ }^{1}$

\section{Resumen}

\begin{abstract}
Este artículo se basa en los resultados obtenidos en dos investigaciones realizadas en la sede Norte de la Universidad de Antioquia, en el marco del énfasis en entrenamiento deportivo en los Niveles IX y X de la Licenciatura en Educación Física, el cual se desarrolló durante los semestres 2011-I y 2011-II.

La premisa utilizada para la realización de las investigaciones fue estructurar las planificaciones deportivas de acuerdo con el método tradicional. Así, los entrenadores-estudiantes verificaron los resultados de una planificación estructurada que cumpla con los principios básicos del entrenamiento deportivo comparando tales resultados con grupos que no realizan planificación. Se realizaron planes de entrenamiento en Yarumal, donde se tomaron cinco grupos de jugadores de fútbol sala, tres para la intervención y dos para el control y en Santa Rosa de Osos, un grupo de Atletismo, en este caso sin grupo control, la planificación utilizada se comparó con grupos control de acuerdo con los dos grupos de edad utilizados, los grupos control siguieron adelantando entrenamientos como parte de su preparación, sin contar con una planificación estructurada; en tanto que a los atletas de Santa Rosa se les planificó conservando la estructura tradicional y enfatizando en las Capacidades Coordinativas. En general, se obtuvieron resultados importantes en los cuatro grupos considerados en las dos modalidades trabajadas de estos dos municipios del Norte del departamento de Antioquia.
\end{abstract}

Palabras claves: Planificación del entrenamiento, planificación tradicional, capacidades condicionales, capacidades coordinativas.

\section{Abstract}

This article is based on the results gotten in two papers that were made in the Antioquia's University in its North Region, on the emphasis of Sports Training in levels IX and X of the Physical Education Under Degree developed during the semesters 2011- I and 2011- II.

The premise used for the making of the researches was giving structures to the Sports Plannings according to the traditional method, in that way the trainers-students verified the results produced by having a wellstructured sports plan that fullfills all the basic principles of sports training and that could produce specific effects, some training plans were done in Yarumal (Antioquia, Colombia), where five groups of hall football players were taken (three for the intervention, and two for the control) and some others in Santa Rosa De Osos (Antioquia, Colombia.) with an athletic group. The planning for the hall football players was modified by ages, and then it was compared with two control groups according to those ages; the control groups continued with their preparation without having with a well-structured sports planning; in contrast to the work prepared for the athletes that was made conserving the traditional structure and focusing in the coordination abilities. In general, important results were obtained from the four groups considered in both modalities worked in those municipalities from the North Region of Antioquia.

Keywords: planning of the training, traditional planning, conditional capacities, coordinative capacities.

Fecha de recepción: 15 de octubre de 2014

Fecha de aprobación: 15 de marzo de 2015

Para citar este artículo:

Agudelo, C.A. (2015). Efectos del entrenamiento estructurado en escolares del norte antioqueño. Lúdica Pedagógica, (21), 153-162.

1 Magíster en Motricidad: Desarrollo Humano por la Universidad de Antioquia, coordinador del pregrado Profesional en Entrenamiento Deportivo, Instituto Universitario de Educación Física Universidad de Antioquia. Correo electrónico: carlosa.agudelo@udea.edu.co 


\section{INTRODUCCIÓN}

En el énfasis de entrenamiento deportivo del programa de Educación Física que se realizó durante 2011, semestres I y II en la sede Norte de la Universidad de Antioquia, se llevaron a cabo dos trabajos de investigación cuantitativa cuya premisa fundamental fue estructurar las planificaciones por el método tradicional y verificar algunos cambios en las capacidades condicionales o coordinativas en los grupos de atletas que fueron intervenidos. En el municipio de Yarumal se trabajó con tres grupos de niños jugadores de fútbol sala entre 8 y 14 años y en Santa Rosa de Osos, con atletas entre 9 y 11 años, las investigaciones consistieron en verificar si las planificaciones realizadas producen cambios significativos en estas poblaciones, para la verificación estadística se utilizó el programa SPSS versión 15, con licencia de la sede Norte de la Universidad de Antioquia.

La asesoría y constante supervisión de la intervención de estos trabajos llevó a que los estudiantes-entrenadores-investigadores realizaran fielmente a lo estructurado los planes de trabajo, previamente adecuados a la edad y la disciplina deportiva.

En cuanto al fútbol sala se seleccionaron para controlar el trabajo la resistencia y la velocidad, consideradas por distintos autores como las capacidades fundamentales de esta disciplina. Estas capacidades determinan el rendimiento en esta modalidad (Riveiro, 2000), ya que supone una lógica interna que da muy poco tiempo para la ejecución de acciones (velocidad). En general, las metodologías que llevan a mejorar la potencia y la velocidad de movimientos son las que llevan a mejorar la capacidad de juego (Pombo, 2007), y esta característica es la que marca realmente diferencia en los tiempos reales de juego, tratando de evitar una alta y negativa influencia de la fatiga (resistencia). La falta de rigor en el entrenamiento de esta modalidad puede disminuir el rendimiento (Prieto, 2006).

La intervención en el trabajo de los jugadores de sala se realizó en ocho microciclos que hicieron parte de la planificación de la etapa general del periodo preparatorio de la macroestructura de planificación tradicional que se implementó para estos tres equipos que compiten en el torneo interno del municipio de Yarumal, estos parámetros de distribución de la programación están muy de acuerdo con la propuesta básica de planificación tradicional (Vasconcelos, 2004), derivada de la obra clásica de Matveév, (1977), que llama a esta primera instancia la primera fase de la creación y desarrollo de la forma deportiva dentro de la concepción clásica de planificación tradicional.

Para los atletas de Santa Rosa de Osos se acentuó el trabajo de las capacidades coordinativas en el periodo competitivo; lo coordinativo tiene tres grandes aplicaciones: en la vida cotidiana, en el aprendizaje motor y en el rendimiento deportivo (Jacob, 1991), se tenía una planificación previamente estructurada por el método tradicional, y con base en el concepto anterior se utilizó lo coordinativo para que incida en el rendimiento deportivo, esto implicó que durante las seis semanas previas a la competencia se llevaran a cabo los estímulos coordinativos en el marco de lo previamente planificado.

El objetivo de implementar tareas coordinativas en esta edad era lograr un mejoramiento de la ejecución de dos de las pruebas utilizadas en juegos departamentales en esta categoría, los lanzamientos, que para el caso de esta categoría está representado por el lanzamiento de la pelota de béisbol y los saltos, en este caso el salto largo con impulso. Se verificaron las mejoras que tal tipo de tareas puede producir en la ejecución de la técnica, en términos de desarrollo motor, la educación motriz infantil se propone facilitar maduración frente al control corporal, permitiendo modificaciones que permitan desarrollar acciones (García, 1999). Para medir las posibles mejoras en la coordinación misma, se aplicó el test de recorrido vienés propuesto en Entrenamiento total (Weineck, 2005), muy semejante a otros que se proponen con el nombre de carrera Boomerang con cajones de plinto (Harre, 1973).

En estas edades consideradas sensibles por excelencia para lo coordinativo hay varias propuestas importantes en los momentos que tienen una mayor sensibilidad en lo condicional, para los estudios referidos se tomó en cuenta la propuesta realizada por Blanco (1994): 
Tabla 1. Fases sensibles de velocidad y resistencia (en años)
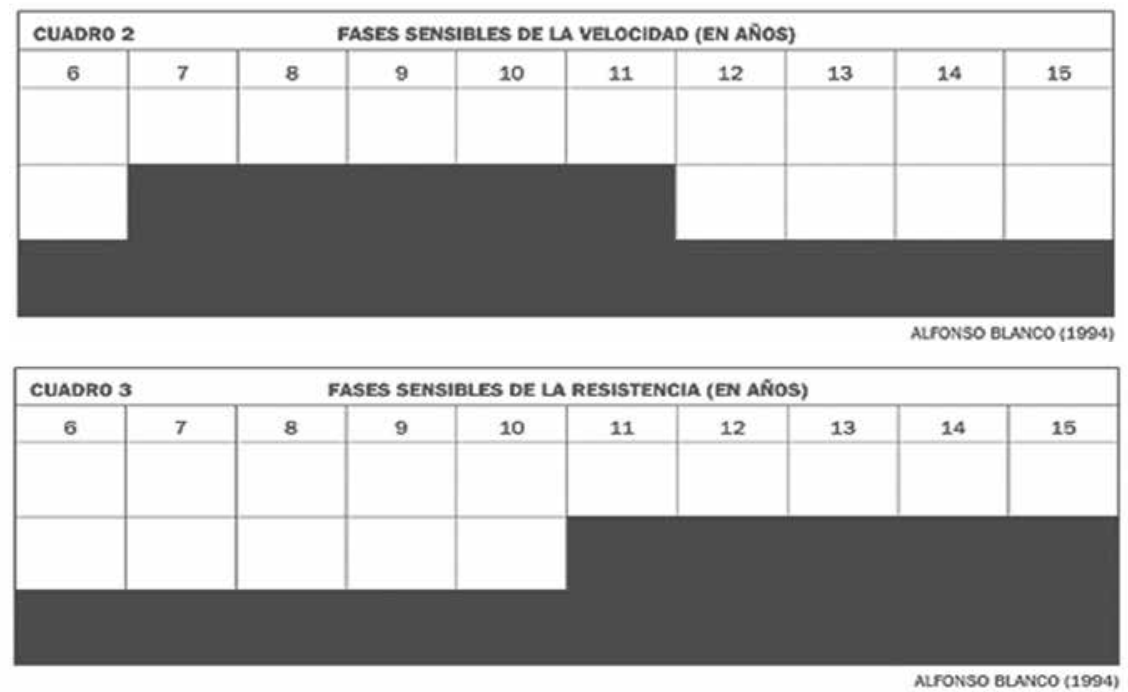

También se tuvieron en cuenta para adaptar las cargas por edad las densidades de carga. Se entiende por densidad de la carga (Grosser, 1990) la relación entre el tiempo de trabajo y el tiempo de recuperación, por ejemplo, 1:2 representa que por cada segundo o minuto trabajado se recupera dos el atleta, en tanto 1:3 representa una proporción de densidad de un minuto de trabajo por 3 de recuperación, y así sucesivamente. Obviamente, las densidades 1:8 (por cada unidad de tiempo de trabajo 8 de recuperación) y mayores fueron utilizadas para el desarrollo de la velocidad en el grupo de 8 a 11 años, en tanto unas densidades un poco menores se usaron para el estímulo de la resistencia en las edades entre 12 y 14 años de edad.

\section{OBJETIVOS}

- Fútbol Sala en Yarumal: analizar los cambios condicionales que produce una etapa de ocho semanas en la planificación tradicional en escolares de fútbol sala.

- Atletismo en Santa Rosa de Osos: analizar los cambios coordinativos que produce una etapa de ocho semanas en la planificación tradicional en escolares de atletismo.

\section{METODOLOGÍA}

\section{Consideraciones éticas}

Para la población intervenida, se realizaron permisos firmados por los acudientes y consentimientos por parte de los sujetos, como lo demandan la declaratoria de Helsinki y la resolución 8430 de 1993 del Ministerio de Salud de Colombia, además se realizó una reunión de padres de familia donde se explicó que la investigación solo pretendía organizar la práctica deportiva que los niños ya tenían como parte de su formación y competencia escolar, en esta reunión con padres de familia, estudiantes en formación y asesor de la práctica de la Universidad se recalcó que la mayor pretensión era organizar, desde una planificación clásica, el sistema de entrenamiento que se iba a aplicar con la ayuda de tablas de control del volumen y la intensidad de las cargas realizadas, así como procurar un mejor orden metodológico en la intervención de los estudiantes que realizaban como entrenadores la intervención.

\section{Instrumentos de medición}

A los datos se les aplicó, con el programa SPSS versión 15 , la prueba de rangos de Wilcoxon, común para los que presentaban una distribución de datos no paramétrica. Esta decisión se debe al bajo número de sujetos que se tiene en el trabajo y por ello se recomienda aplicar directamente pruebas no paramétricas para tener una mayor probabilidad de resultados reales (Rubio, 2012). La metodología consistió en realizar las mismas pruebas, a la misma hora del día, antes (pretest) y después (postest) de las respectivas intervenciones.

Los datos se obtuvieron con las siguientes pruebas como instrumentos de medición: 
Pruebas aplicadas a jugadores de fútbol sala de Yarumal

Resistencia, a través de la ecuación de Bruno Balke, que permite aplicar diferentes protocolos por edad, para este trabajo se definió así: 5 minutos de 8-11 años y 12 minutos de 12-14 años, respaldados en las variaciones realizadas a tal valoración (Heyward, 2008), donde la variable a medir es la distancia recorrida por los sujetos, tal medición se realizó con el mismo parámetro y en el mismo escenario tanto en el pretest como en el postest.

Velocidad, a través de la prueba de 20 metros lanzados, cuyo protocolo está descrito en el manual de pruebas físicas de Coldeportes Nacional (Jauregui y Ordóñez, 1993). Como se conoce, los protocolos deben ser específicos por deporte, por ello en estos trabajos se trató de respetar tal indicación tanto en los controles como en las tareas a aplicar durante la intervención, así como en la planeación y selección de las componentes condicionales de las cargas (Brown, 2007).

Santa Rosa de Osos

Para medir los tiempos de las fases de la técnica, se utilizó el programa KINOVEA, que permitió tener una herramienta valiosa al dividir los videos de los atletas a secuencias de fotogramas cada 0,04 segundos y con base en estos poder calcular los tiempos de ejecución de cada una de las fases de las dos técnicas evaluadas. Y para medir el índice de coordinación se utilizó el test vienés tal y como se recomienda en el texto de Weineck (2005), retomado a su vez de Warwitz, consistente en tomar el tiempo en un recorrido que se ilustra así:

Figura 1. Recorrido de coordinación vienés de Warwitz.

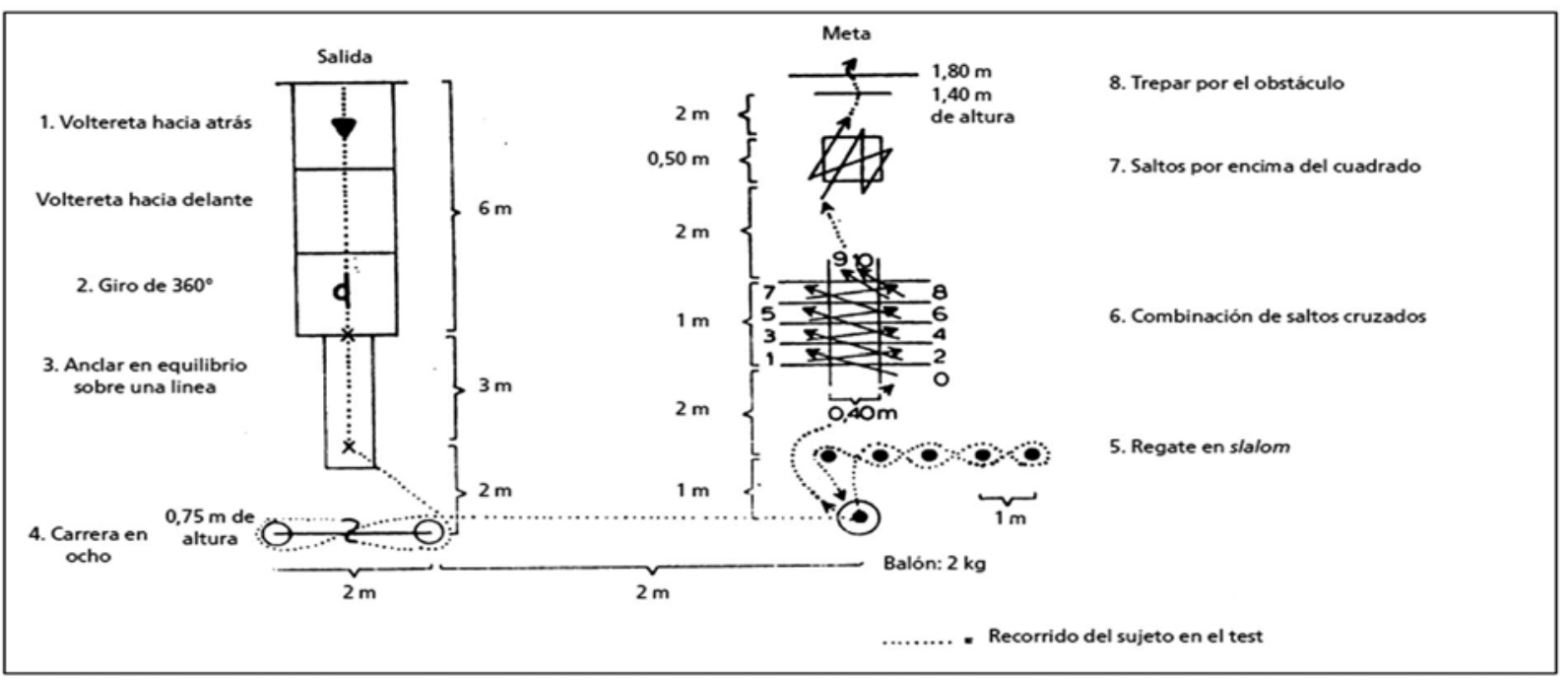

\section{Población}

Fútbol Sala en Yarumal

En este trabajo se tomó un total de cinco grupos de jugadores de fútbol sala para poder realizar el trabajo de investigación, tres para la intervención y dos de control, los grupos de intervención estuvieron divididos así: dos grupos de 8 a 11 años y un grupo de 12 a 14 .

En el grupo de 8 a 11 años se analizaron los dos grupos y no se encontraron diferencias significativas entre ellos, por tanto, para asuntos metodológicos, tales datos se juntaron para ser analizados contra el grupo control de tal edad, ya que el plan de intervención fue el mismo, se diseñó con los dos entrenadores, puesto que entrenaban tanto el mismo número de veces por semana como el mismo tiempo por sesión.
De 8 a 11 años es más susceptible estimular la velocidad, que depende más de lo coordinativo y lo general del desarrollo motor. Blanco (1994) plantea que de 7 a 11 años es la edad de mayor incidencia en el desarrollo de la velocidad, la que denomina fase sensible por cuanto de 12 a 14 ya se puede iniciar con trabajos que favorezcan más la resistencia.

Los profesores del grupo de 8 a 11 años fueron los estudiantes John Jairo Ceballos Vélez y Daniel Arturo Alcaraz Fernández, el estudiante que orientó el grupo de 12 a 14 años fue Hernán Alberto Echavarría Arbeláez, los grupos pertenecían a instituciones educativas de Yarumal que compiten por representar al municipio en el proceso de zonales y de juegos departamentales así: 
Grupos de intervención de 8 a 11 años:

- Escuela María Auxiliadora, practicante Jhon Jairo Ceballos Vélez

- Colegio Católico, practicante Daniel Arturo Alcaraz Fernández

- Grupo control: Normal Superior La Merced

Grupo de Intervención de 12 a 14 años:

- San Luis, practicante Hernán Alberto Echavarría Arbeláez

- Grupo control: Institución Educativa de María

Atletismo en Santa Rosa de Osos

Este estudio es prácticamente de caso, ya que los sujetos seleccionados fueron solo cuatro, dos de cada género, se les aplicó el plan de trabajo y se hizo un pretest y postest, donde se tomaron las marcas registradas en los dos momentos: antes y después de las seis semanas de intervención, tales pruebas se filmaron y se analizaron con el programa KINOVEA 18.15, para establecer qué ocurrió en las fases de los movimientos y verificar las posibles causas del comportamiento de las marcas.

\section{Variable independiente: planificaciones}

Se utilizó un plan de trabajo que definió la etapa de preparación física general del periodo preparatorio en ocho semanas compuesto por tres mesociclos, un mesociclo entrante de dos microciclos (semanas), un mesociclo desarrollador de tres semanas y un mesoestabilizador de tres semanas, la idea consistió en que las cargas de velocidad en 8-11 años y de resistencia en 12-14 años se definieran muy concretamente, estas diferencias están dadas principalmente por los tiempos de aplicación de las cargas, la dosificación de tales cargas, especialmente en la medición de la densidad de la carga, y en la latencia adecuada para cada edad. Tienen en común unos criterios unificados en sus tablas de volumen e intensidad, según la planificación por modelamiento (Agudelo, 2012), el tiempo de duración de los estímulos y el número de sesiones semanales programadas, el plan grafico en ambas edades presenta la distribución del trabajo en tres mesociclos, que componen la etapa de preparación física general del periodo preparatorio de ocho semanas comprendido entre el primero de agosto y el 25 de septiembre de 2011, como parte del plan general estructurado para el segundo semestre de 2011 por los estudiantes-entrenadores-investigadores.

Planes utilizados para la intervención en jugadores de fútbol sala en Yarumal

Tabla 2. Plan gráfico de la edad de 8 a 11 años.

\begin{tabular}{|c|c|c|c|c|c|c|c|c|c|}
\hline \multicolumn{2}{|c|}{ MESES. } & \multicolumn{4}{|c|}{ AGOSTO. } & \multicolumn{4}{|c|}{ SEPTIEMBRE. } \\
\hline \multirow{2}{*}{\multicolumn{2}{|c|}{ FECHAS. }} & 1 & 8 & 15 & 22 & 29 & 5 & 12 & 19 \\
\hline & & 7 & 14 & 21 & 28 & 4 & 11 & 18 & 25 \\
\hline \multicolumn{2}{|c|}{ PERIODO. } & \multicolumn{8}{|c|}{ PREPARATORIO. } \\
\hline \multicolumn{2}{|c|}{ ETAPAS. } & \multicolumn{8}{|c|}{ PREPARACIÓN FÍSICA GENERAL GENERAL. } \\
\hline \multicolumn{2}{|c|}{$\begin{array}{l}\text { MESOCICLOS. } \\
\text { MICROCICLOS. }\end{array}$} & \multicolumn{2}{|c|}{ ENTRANTE. } & \multicolumn{3}{|c|}{ BASICO DESARROLLADOR. } & \multicolumn{3}{|c|}{ ESTABILIZADOR. } \\
\hline & CICLOS. & 1 & 2 & 3 & 4 & 5 & 6 & 7 & 8 \\
\hline \multicolumn{2}{|c|}{ VOLUMEN. } & 5 & 5 & 5 & 5 & 5 & 4 & 5 & 3 \\
\hline \multicolumn{2}{|c|}{ INTENSIDAD. } & 2 & 2 & 3 & 3 & 3 & 4 & 4 & 3 \\
\hline \multicolumn{2}{|c|}{ PORCENTAJE. } & $299 \mathrm{~min}$ & $299 \mathrm{~min}$ & $299 \mathrm{~min}$ & $275 \mathrm{~min}$ & $290 \mathrm{~min}$ & $265 \mathrm{~min}$ & $285 \mathrm{~min}$ & $230 \mathrm{~min}$ \\
\hline \multirow{4}{*}{$\begin{array}{l}\text { DIAS DE } \\
\text { TRABAJO }\end{array}$} & Martes & 1 & 1 & 1 & 1 & 1 & 1 & 1 & 1 \\
\hline & Miercoles & 1 & 1 & 1 & 1 & 1 & 1 & 1 & 1 \\
\hline & Jueves & 1 & 1 & 1 & 1 & 1 & 1 & 1 & 1 \\
\hline & sabado-partido & 1 & 1 & 1 & 1 & 1 & 1 & 1 & 1 \\
\hline \multicolumn{2}{|c|}{ MINUTOS DEL TRABAJO FISICO } & $150 \mathrm{~min}$ & $150 \mathrm{~min}$ & $150 \mathrm{~min}$ & $138 \mathrm{~min}$ & $145 \mathrm{~min}$ & $90 \mathrm{~min}$ & $100 \mathrm{~min}$ & $80 \mathrm{~min}$ \\
\hline \multirow{2}{*}{\multicolumn{2}{|c|}{ VELOCIDAD. }} & & & \begin{tabular}{|c|} 
INTERVALOS. \\
15 MIN. 1:2 X5
\end{tabular} & \begin{tabular}{|c|} 
INTERVALOLS. \\
15 MIN. 1:2 X5
\end{tabular} & $\begin{array}{l}\text { INTERVALOS. } \\
\text { 15 MIN.1:2 X5 }\end{array}$ & \begin{tabular}{|c|} 
INTERVALOS. 15 \\
MIN. 1:2 $\times 5$ \\
CON BALON \\
PEGADA A \\
PORTERIA. \\
\end{tabular} & \begin{tabular}{|} 
INERVALOS. 20 \\
MIN. $1: 3 \times 5$ \\
CON BALON. \\
JUEGO $2 \times 2$
\end{tabular} & $\begin{array}{l}\text { INTERVALOS. } \\
20 \text { MIN 1:3 X5 } \\
\text { CON BALON. } \\
\text { JUEGO 1X1. }\end{array}$ \\
\hline & & $\begin{array}{l}\text { REPETICIONES. } \\
30 \text { MIN. 1:5 XS } \\
\text { REP }\end{array}$ & $\begin{array}{l}\text { REPETICIONES. } \\
30 \text { MIN 1:5 XS } \\
\text { REP }\end{array}$ & \begin{tabular}{|c|} 
REPETICIONES. \\
30 MIN. $1: 5$ XS \\
REP
\end{tabular} & \begin{tabular}{|c|} 
REPEETCIONES. \\
28 MIN. $1: 6$ X4 \\
REP
\end{tabular} & $\begin{array}{l}\text { REPETICIONES. } \\
25 \text { MIN. 1:4 XS } \\
\text { REP }\end{array}$ & \begin{tabular}{|c|} 
REPETCIONESS \\
30 MIN. CON \\
BALON 1:5 X5 \\
PASE RASTRERO \\
CON BORDE \\
INTERNO. EN \\
MOVIMIENTO \\
\end{tabular} & \begin{tabular}{|c|} 
REPETICIONES. \\
30 MIN. CON \\
BALON $1: 5 \times 5$. \\
CONTROL CON \\
PLANTA DE PIE \\
+ PEGADA A \\
PORTERIA. \\
\end{tabular} & $\begin{array}{c}\text { REPETICIONES. } \\
20 \text { MIN. CON } \\
\text { BALON } 1: 3 \text { XS. } \\
\text { TRENZA. }\end{array}$ \\
\hline \multicolumn{2}{|c|}{ TABLA DE VOLUMEN } & & & \multicolumn{2}{|c|}{ TABLA DE INTENSIDAD } & & & & \\
\hline 5 & 270 MIN O MAS & & & 5 & 4o MAS & & & & \\
\hline 4 & $240-269$ & & & 4 & 3 & & & & \\
\hline 3 & $210-239$ & & & 3 & 2 & & & & \\
\hline 2 & $180-209$ & & & 2 & 1 & & & & \\
\hline 1 & MENOS DE 180 & & & 1 & 0 & & & & \\
\hline
\end{tabular}


Los métodos utilizados fueron variables o invariables, con la densidad descrita en cada caso, y con el número de tareas de dificultad realizada por microciclo como criterio de intensidad y el total de minutos de trabajo de la semana como criterio para el volumen, según el modelamiento (Agudelo 2012), como se trata del periodo preparatorio se puede observar que los volúmenes son de cinco en casi todos los micros y en ningún caso baja de tres.
El plan de trabajo de los jugadores de sala de 12 a 14 años tiene diferencias marcadas en la dosificación de las tareas que se consideran de resistencia y de velocidad, que son las que se estarán evaluando, ya que la edad es un factor determinante (Navarro, 2004) en la forma como debe ser aplicada la carga cumpliendo con aquel parámetro de buscar el deporte para el niño y no acomodar el niño al deporte (Durand, 1987).

Tabla 3. Plan gráfico aplicado al grupo con edades de 12-14 años.

\begin{tabular}{|c|c|c|c|c|c|c|c|c|c|}
\hline \multicolumn{2}{|c|}{ MESES. } & \multicolumn{4}{|c|}{ AGOSTO. } & \multicolumn{4}{|c|}{ SEPTIEMBRE. } \\
\hline \multirow{2}{*}{\multicolumn{2}{|c|}{ FECHAS. }} & 1 & 8 & 15 & 22 & 29 & 5 & 12 & 19 \\
\hline & & 7 & 14 & 21 & 28 & 4 & 11 & 18 & 25 \\
\hline \multicolumn{2}{|c|}{ PERIODO. } & \multicolumn{8}{|c|}{ PREPARATORIO. } \\
\hline \multicolumn{2}{|c|}{ ETAPAS. } & \multicolumn{8}{|c|}{ PREPARACIÓN FISICA GENERAL GENERAL } \\
\hline \multirow{2}{*}{\multicolumn{2}{|c|}{$\begin{array}{l}\text { MESOCICLOS. } \\
\text { MICROCICLOS. }\end{array}$}} & \multicolumn{5}{|c|}{ BASICO DESARROLADOR. } & \multicolumn{3}{|c|}{ ESTABILIZADOR. } \\
\hline & & 1 & 2 & 3 & 4 & 5 & 6 & 7 & 8 \\
\hline \multicolumn{2}{|c|}{ VOLUMEN. } & 5 & 5 & 4 & 4 & 4 & 4 & 4 & 4 \\
\hline \multicolumn{2}{|c|}{ INTENSIDAD. } & 2 & 2 & 2 & 2 & 2 & 2 & 3 & 3 \\
\hline \multicolumn{2}{|c|}{ PORCENTAUE. } & 300 & 300 & 290 & 290 & 290 & 270 & 270 & 270 \\
\hline \multirow{4}{*}{$\begin{array}{l}\text { DIAS DE } \\
\text { TRABAIO }\end{array}$} & Martes & 1 & 1 & 1 & 1 & 1 & 1 & 1 & 1 \\
\hline & Miercoles & 1 & 1 & 1 & 1 & 1 & 1 & 1 & 1 \\
\hline & Jueves & 1 & 1 & 1 & 1 & 1 & 1 & 1 & 1 \\
\hline & sabado-partido & 1 & 1 & 1 & 1 & 1 & 1 & 1 & 1 \\
\hline \multicolumn{2}{|c|}{ MINUTOS DEL TRABAO FISICO } & $150 \mathrm{~min}$ & $150 \mathrm{~min}$ & $150 \mathrm{~min}$ & $138 \mathrm{~min}$ & $145 \mathrm{~min}$ & $90 \mathrm{~min}$ & $100 \mathrm{~min}$ & $80 \min$ \\
\hline \multirow{2}{*}{\multicolumn{2}{|c|}{ RESISTENCIA. }} & $\begin{array}{l}\text { VARAABLE. } \\
\text { 90 MIN. } \\
\text { CARRERA } \\
6: 2 \times 4 \text { RCP }\end{array}$ & $\begin{array}{c}\text { VARAABLE. 90 } \\
\text { MIN. CARRERA } \\
6: 3 \times 3 \text { REP }\end{array}$ & $\begin{array}{c}\text { VARLABLE. } \\
\text { 80 MIN. } \\
\text { CARRERA 5:3 X } \\
\text { 5 VECES } 2 \text { ON EL } \\
\text { MICRO }\end{array}$ & \begin{tabular}{|c|} 
VARIABLE. 60 \\
MIN. CARRERA \\
X3 DIAS 3:2 4 \\
REP \\
\end{tabular} & $\begin{array}{c}\text { VARIABLE. } 60 \\
\text { MIN. CARRER } \\
\times 3 \text { DIAS 3:2 X4 } \\
\text { RLP }\end{array}$ & $\begin{array}{c}\text { VARIABLE. } 30 \\
\text { MIN. CON } \\
\text { BALON ZIG-ZAG } \\
\text { 15 XS RLP }\end{array}$ & \begin{tabular}{|} 
VARIABLE. 30 \\
MIN. CON \\
BALON \\
CONTRO AERCO \\
$1: 5 \times 5$ REP
\end{tabular} & $\begin{array}{c}\text { VAR. } 30 \text { MIN. } \\
\text { CON BALON } \\
\text { CONTROL CON } \\
\text { BORDE INTRNO } \\
\text { +PASES1:5 X5 } \\
\text { RCP } \\
\end{array}$ \\
\hline & & \begin{tabular}{|c} 
INVARIABLE. 30 \\
MIN. \\
CARRERA CADA \\
2 DIAS 15 MIN \\
\end{tabular} & $\begin{array}{c}\text { IN ARIABLC. } 30 \\
\text { MIN. } \\
\text { CARRERA CADA } \\
2 \text { DIAS } 15 \text { MIN } \\
\end{array}$ & $\begin{array}{c}\text { INVARIABLE. } 36 \\
\text { MIN. CARRERA } \\
\text { CADA DIA 12 } \\
\text { MIN }\end{array}$ & $\begin{array}{c}\text { INVARIABLE25 } \\
\text { MINCARRERA } \\
12 \text { MIN. CADA2 } \\
\text { DIAS }\end{array}$ & $\begin{array}{c}\text { INVARIABLE } 35 \\
\text { MIN. CARRERA } \\
\begin{array}{c}12 \text { MIN CADA } \\
\text { DIA }\end{array} \\
\end{array}$ & $\begin{array}{l}\text { INVARIABLE. } 15 \\
\text { MIN. CON } \\
\text { BALON }\end{array}$ & $\begin{array}{c}\text { INVARIABLE. 20 } \\
\text { MIN. CON } \\
\text { BALON }\end{array}$ & $\begin{array}{l}\text { INVARIABLE } 10 \\
\text { MIN. CONTROL } \\
\text { CON PLANTA DE } \\
\text { PIE. }\end{array}$ \\
\hline & & & & & & & & & \\
\hline \multicolumn{2}{|c|}{ TABLA DE VOLUMEN } & & & \multicolumn{2}{|c|}{ TABLA DE INTENSIDAD } & & & & \\
\hline 5 & $300 \mathrm{MIN}$ O MAS & & & 5 & $60 \mathrm{MAS}$ & & & & \\
\hline 4 & $270-299$ & & & 4 & 405 & & & & \\
\hline 3 & $240-269$ & & & 3 & 3 & & & & \\
\hline 2 & $210-239$ & & & 2 & 102 & & & & \\
\hline 1 & MENOS DE 210 & & & 1 & 0 & & & & \\
\hline
\end{tabular}

Plan utilizado para los atletas en Santa Rosa de Osos

A continuación un ejemplo del plan que hizo parte del periodo competitivo y mantuvo estímulos constantes de las capacidades coordinativas en las seis semanas de intervención:

Tabla 4

\begin{tabular}{|c|c|c|c|c|c|}
\hline $\begin{array}{c}19 \text { al } 25 \mathrm{de} \\
\text { septiembre/DÍAS }\end{array}$ & LUNES & MARTES & MIÉRCOLES & JUEVES & VIERNES \\
\hline PARTE INICIAL & $\begin{array}{l}\text { 10' trote. Juego: } \\
\text { el mensajero }\end{array}$ & $\begin{array}{l}\text { Juego: ¿quién } \\
\text { salta más? }\end{array}$ & \multirow{3}{*}{ DESCANSO } & $\begin{array}{l}\text { 10' trote. Juego: sal- } \\
\text { tar en círculos }\end{array}$ & $\begin{array}{c}\text { Juego: Saltar } \\
\text { con objetos }\end{array}$ \\
\hline PARTE CENTRAL & $\begin{array}{l}\text { Técnica + capaci- } \\
\text { dades de orien- } \\
\text { tación: despla- } \\
\text { zamiento indivi- } \\
\text { dual y parejas en } \\
\text { varias direcciones }\end{array}$ & $\begin{array}{l}\text { Técnica + capa- } \\
\text { cidades de } \\
\text { reacción: pisar } \\
\text { al compañero }\end{array}$ & & $\begin{array}{l}\text { Técnica + capacida- } \\
\text { des de ritmo: sal- } \\
\text { tos con cada pierna }\end{array}$ & $\begin{array}{c}\text { Técnica }+c \\
\text { capacidades } \\
\text { de equili- } \\
\text { brio: parada } \\
\text { en talones y } \\
\text { hacer varias } \\
\text { actividades }\end{array}$ \\
\hline PARTE FINAL & Estiramiento & Estiramiento & & Estiramiento & Estiramiento \\
\hline
\end{tabular}


Son seis semanas de trabajo con este tipo de intervención coordinativa, las otras cinco semanas en que se realizó el trabajo fueron:

26 de septiembre al 2 de octubre, 3 al 9 de octubre, 10 al 16 de octubre, 17 al 23 de octubre y 24 al 30 de octubre.

Como se observa en la parte central, de los 24 entrenamientos se tiene propuesto el desarrollo de las capacidades coordinativas, ya sea acompañado de otras tareas técnicas o no, lo que indiscutiblemente constituye una novedad de esta planificación tradicional con una distribución diferente de la carga.

\section{Hipótesis}

Hipótesis nula (Ho): una etapa del entrenamiento que se realice bajo un plan estructurado no produce cambios importantes de tipo condicional/coordinativo en la edad propuesta para la modalidad (fútbol sala o atletismo, según cada caso).
Hipótesis alterna (Ha): una etapa realizada con un plan estructurado produce cambios importantes de tipo condicional/coordinativo en la edad propuesta para la modalidad (fútbol sala o atletismo, según cada caso).

\section{RESULTADOS}

A pesar de tener referentes investigativos que apuntan a que en jugadores jóvenes de fútbol es más importante entrenar la técnica que lo condicional (Katis y Kellis, 2009), el presente estudio quería verificar qué ocurre en lo condicional cuando se estructura una planificación para el caso del fútbol sala.

\section{Resultados de fútbol sala en Yarumal}

Los resultados obtenidos en la prueba antes y después de la intervención en las variables que dieron un mejor valor p son: cuando se compararon los grupos control e intervención, se vieron como significativos los cambios en velocidad de 8-11 años y en resistencia en el grupo de 12-14, con los siguientes resultados.

Tabla 5. Resultados fútbol sala en Yarumal según rango de edad.

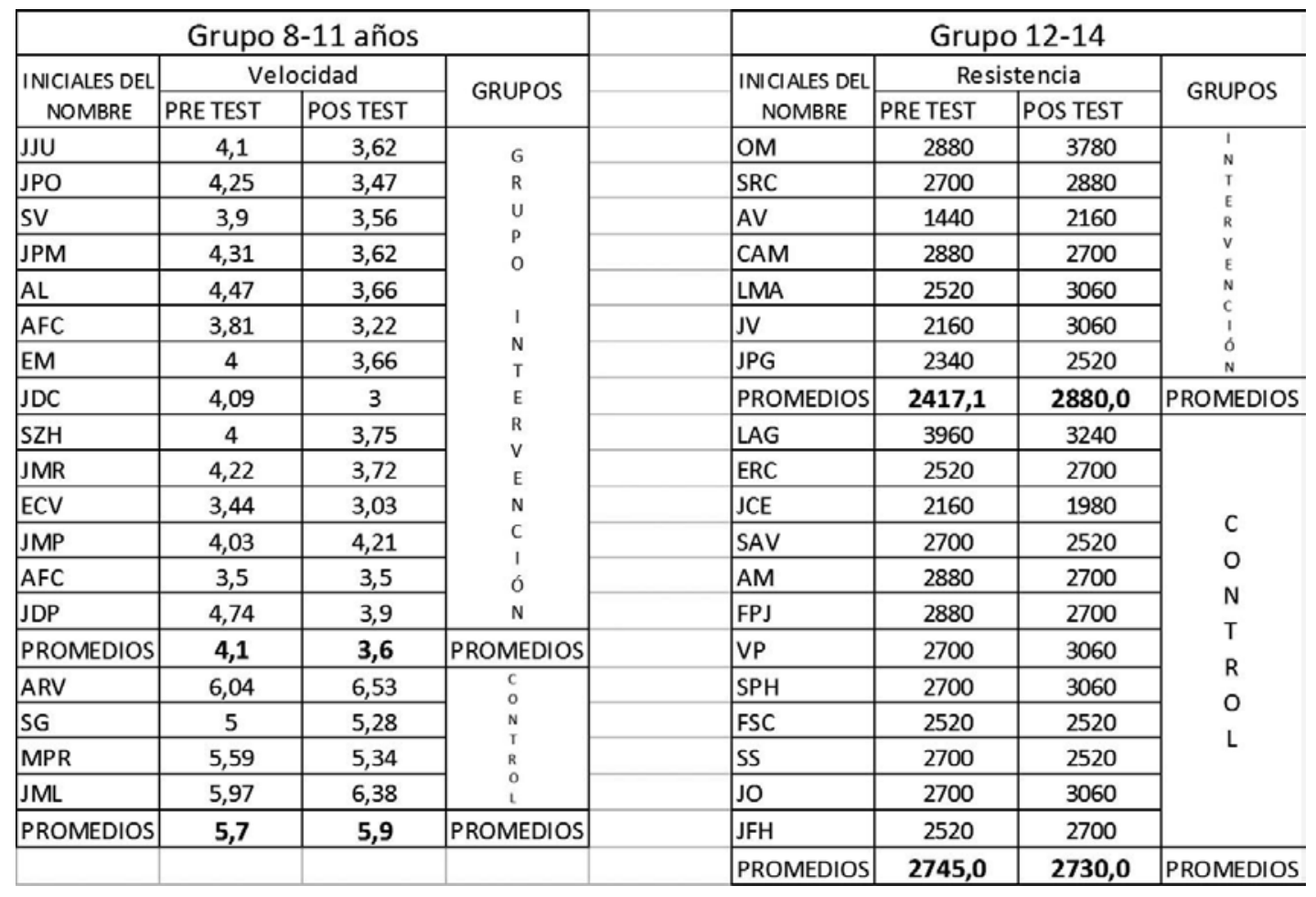

Las tablas y valores estadísticos se encuentran expuestos en el libro que generó el reseñado trabajo de grado y que publicaron los autores, por ello no se considera pertinente presentarlos en este artículo que quiere ver desde otra óptica los fenómenos ocurridos (Echavarría, Alcaraz y Ceballos, 2012). 


\section{Resultados en atletismo en Santa Rosa de Osos}

Para esta modalidad, el estudiante-entrenador es el desarrollador del municipio de la modalidad, el profesor Robinson Alexander Roldán Arroyave, quien dirige los semilleros de Santa Rosa donde se seleccionan los sujetos para representar en los zonales y en los juegos departamentales en la modalidad de atletismo.

El estudio consistió en tomar los cuatro sujetos que clasificaron a la fase final, aplicarles un estímulo en capacidades coordinativas y verificar si la aplicación de estos estímulos durante las seis semanas previas a la competencia departamental en este tipo de capacidades produce algún efecto en la marca de las pruebas que deben realizar en salto y lanzamiento como parte de las pruebas que son incluidas en las competencias de la edad escolar. El ámbito coordinativo engloba los movimientos relacionados con la calidad de la ejecución (Tejada y Peñas, 2003) y que, como se reconoce, depende del desarrollo motor, del que se puede afirmar que está vinculado con una serie de leyes biológicas, pero también de las interacciones susceptibles de estimulación y aprendizaje (Cobos, 1995), en este estudio de casos se pretende aprovechar que los sujetos están en una edad que se considera sensible para el desarrollo coordinativo y se puede pensar que es posible lograr un mejor nivel puntual en estas acciones deportivas del atletismo.

El pretest y postest de los cuatro sujetos consistió en lo siguiente, para cada atleta:

- Realiza tres saltos.

- Realiza tres lanzamientos.

- Se promedia como resultado del test los tres resultados.

- Se analizan las fases de cada uno de los mejores intentos, la fases del mejor salto y las fases del mejor lanzamiento.

- Se ejecuta el test vienés general de coordinación.

Tabla 6. Resultados Santa Rosa de Osos.

\begin{tabular}{|c|c|c|c|c|c|c|c|c|c|c|}
\hline \multirow{2}{*}{ Sujeto } & \multicolumn{2}{|c|}{ PROM Saltos } & \multicolumn{2}{|c|}{ PROM Lanza } & \multicolumn{2}{|c|}{ Fase Vuelo } & \multicolumn{2}{|c|}{ Fase Contacto } & \multicolumn{2}{|c|}{ TEST VIENES } \\
\hline & pretest & postest & pretest & postest & pretest & postest & pretest & postest & pretest & postest \\
\hline VP & 3,30 & 3,30 & 26,90 & 26,10 & 0,48 & 0,50 & 0,16 & 0,10 & 57,02 & 56,56 \\
\hline MQ & 3,40 & 3,30 & 26,10 & 27,80 & 0,48 & 0,50 & 0,16 & 0,14 & 63,46 & 57,22 \\
\hline $\mathrm{CH}$ & 2,30 & 2,50 & 18,20 & 22,50 & 0,40 & 0,43 & 0,28 & 0,20 & 70,36 & 67,40 \\
\hline $\mathrm{GU}$ & 2,70 & 2,90 & 18,50 & 22,90 & 0,20 & 0,42 & 0,20 & 0,18 & 85,82 & 64,41 \\
\hline PROM. & 2,93 & 3,00 & 22,43 & 24,83 & 0,39 & 0,46 & 0,20 & 0,16 & 69,17 & 61,40 \\
\hline
\end{tabular}

Estos resultados muestran una ostensible mejora en la longitud de los saltos y los lanzamientos, además de una disminución en el tiempo de ejecución del test vienés, lo cual permite pensar que haber incluido en la planificación tradicional las cargas coordinativas fue de gran beneficio, al punto que se logró por primera vez en la historia de los Juegos Escolares Departamentales medallas para Santa Rosa en las categorías infantiles de atletismo, ya que las deportistas (MQ) y (VP), como se observó en el boletín oficial de juegos escolares 2011 de Indeportes Antioquia, lograron marcas superiores a las del postest y medallas.

Sin embargo, al aplicar el sistema SPSS para valorar qué tan significativo fue el cambio, no se obtuvieron valores relevantes, lo que permite afirmar que, si bien estadísticamente los cambios no fueron significativos, se ve un incremento en los promedios que, a pesar de no ser estadísticamente significativo, fue suficiente para el logro deportivo alcanzado.
Tabla de valores de la prueba de Wilcolxon en SPSS

Con los datos anteriores, pero ahora tratados según las recomendaciones de Rubio (2012), se obtienen los siguientes resultados de las variables analizadas que resulta más importante comparar:

\begin{tabular}{|c|c|c|c|}
\hline Variable evaluada & GRUPO & P & SIG. \\
\hline Velocidad & $9-11$ años Yarumal & 0,002 & $* *$ \\
\hline Resistencia & $12-14$ años Yarumal & 0,1 & - \\
\hline Test vienés & Atletismo Santa Rosa & 0,068 & - \\
\hline
\end{tabular}

Se debe interpretar como que solo se debe aceptar la hipótesis alterna para el caso de la velocidad en los niños de 9 a 11 años de fútbol de salón en Yarumal y que posiblemente los resultados de significancia obtenido se deben no tanto a la intervención practicada, sino a retrocesos o asuntos relacionados con los grupos control. 


\section{CONCLUSIONES}

Es muy importante resaltar la obtención de buenos resultados cuando se verifican los obtenidos luego de realizar unas planificaciones bien ajustadas en planes de niños entre 8 y 14 años en el norte del departamento de Antioquia, lo cual viene a corroborar que si se planea con métodos tradicionales, teniendo en cuenta algunos aspectos relevantes de la planificación, los resultados pueden llegar a ser muy positivos, aun en estas edades que, como se sabe, se caracterizan por constituir muchos de sus grupos o semilleros con base en programas generales. En conclusión, los trabajos investigativos que originan el presente artículo inducen a pensar que puede llegar a ser preferible en algunos casos tener planes estructurados y no programas generales en estas edades.

De manera puntual, en estos trabajos investigativos se lograron mejorar de forma estadísticamente significativa las capacidades condicionales de escolares que practican fútbol de salón en el municipio de Yarumal, concretamente la velocidad entre los que tienen 8 y 11 años, lo que coincide con las edades de latencia propuestas por Blanco (1994), en tanto la resistencia en estas edades no mejoró sin importar si se tiene o no un plan estructurado. También se encontró que, al comparar contra un grupo control la resistencia en el grupo de 12 a 14 años, también mejora, ratificando las latencias ya mencionadas (Blanco, 1994); por tanto, en el caso de los grupos de Yarumal se toma la hipótesis alterna solo para la velocidad en la edad de 8 a 11 años y para la resistencia, en la de 11 a 14. También se acepta la hipótesis nula para la velocidad de 12 a 14 años y para resistencia de 8 a 11 años.

Para el caso de Santa Rosa, se acepta la hipótesis nula, ya que no hay cambios estadísticamente significativos, pero se logró, además de un sonoro triunfo deportivo inédito para el Municipio, un mejoramiento en los promedios de las marcas de los atletas: tanto en la pruebas de lanzamiento como en la de salto largo, así como en la evaluación del test vienés que permite medir lo coordinativo.

Si bien con los grupos de Yarumal se trabajaron los efectos sobre las capacidades condicionales y en el grupo de Santa Rosa, en las capacidades coordinativas, se puede decir que la metodología de planificar y controlar el entrenamiento deportivo teniendo en cuenta los principios fundamentales de la planificación y las necesidades de la lógica interna del deporte (principio de individualización) pueden constituir un aspecto importante que permite afirmar que se debe definir muy claramente qué se debe controlar para desarrollar y realizar cada tipo de planificación.

También se puede afirmar desde los resultados obtenidos y para estos grupos concretos que los jugadores de fútbol sala entre 8 y 11 años, si entrenan de forma estructurada, mejoran más fácilmente su velocidad, mientras la resistencia no varía en función de estructurar el entrenamiento y entre 12 y 14 años ocurre lo contrario, siendo más factible mejorar la resistencia con planes estructurados con menos influencia sobre la velocidad.

Igualmente, cuando se aplican protocolos estadísticos considerando que las poblaciones no obedecen a condiciones de normalidad, lo que asegura un resultado más confiable, se encuentra que la única capacidad que presenta cambios prácticamente indiscutibles es la velocidad en niños de 8 a 11 años, por tanto, la conclusión más contundente de esta comparación tiene que ver con la recomendación de estructurar trabajos de densidades grandes en edades de 8 a 11 años con una certeza importante de incidencia en mejoras de la velocidad $(p=0,002)$, tal valor da para una valoración del efecto altamente significativa.

La planificación tradicional sigue siendo la más recomendada para las categorías infantiles y juveniles y se recomienda en el entrenamiento de estas categorías seguirla sin tener temor de no obtener rápidos resultados, ya que se está formando deportistas hacia un futuro; lo que se realizó en estos dos trabajos va en esta dirección, verificar que en la medida en que se planifique de una manera estructurada y se controlen las variables adecuadas, la planificación tradicional produce excelentes beneficios en la iniciación y formación deportiva y posiblemente no se tiene la necesidad de abandonar tal paradigma de planificación en los procesos en estas etapas de la vida deportiva.

Se verificó en el presente trabajo que los paradigmas de tablas de control de volumen e intensidad que se proponen en el modelamiento (Agudelo, 2012) tienen una oportuna incidencia para programar de forma tradicional tales componentes de carga en estas edades. 


\section{REFERENCIAS BIBLIOGRÁFICAS}

Agudelo, C. (2012). Planificación del Entrenamiento Deportivo por Modelamiento. Armenia: Kinesis.

Blanco, A. (1994).Temario de oposición segundaria. Madrid: INDE Publicaciones.

Brown, L. y Ferrigno, V. (2007). Entrenamiento de velocidad, agilidad y rapidez. Barcelona: Editorial Paidotribo.

Cobos, P. (1995). El desarrollo psicomotor y sus alcances. Madrid: Editorial Pirámide.

Durand, M. (1987). El niño y el deporte. Editorial Paidós.

Echavarría, H., Alcaraz, D. y Ceballos, J. (2012). Efecto de un plan de entrenamiento en Fútbol Salón. Editorial Académica Española.

Grosser, M. (1990). Alto Rendimiento deportivo: planificación y desarrollo. Barcelona: Editorial Martínez Roca

Harre, D. (1973). Teoría del Entrenamiento Deportivo. La Habana: Editorial Científico Técnica.

Heyward, V. (2008). Evaluación de la Aptitud Física y prescripción del ejercicio. Bogotá: Panamericana.

Jacob, F. (1991). Función e importancia de las cualidades coordinativas. Revista Stadium. (147) 36-40.

Jauregui, G. y Ordóñez, O. (1993). Aptitud Física: pruebas estandarizadas en Colombia. Bogotá: Editorial Nueva Ley.
Katis, A. y Kellis, E. (2009). Effects of small-sided games on physical conditioning and performance in young soccer players. Journal of Sports Science and Medicine, 8, 374-380.

Matveév, L. (1977). Periodización del Entrenamiento Deportivo. Madrid: Instituto Nacional de Educación Física.

Navarro F. (2004). Entrenamiento adaptado a los jóvenes. Revista Educación y Deporte, 335, 61-80.

Pombo, F. (2007). La velocidad de las piernas en el fútbol sala. Revista profesional de Fútbol y Fútbol, 3.

Prieto, I. (2006). Las capacidades condicionales en el joven jugador de fútbol sala. Revista digital efdeportes. com, 99, 1-1.

Riveiro, J. (2000). La preparación física del fútbol sala. Sevilla: Editorial Wanceulen.

Rubio Hurtado, M. J. y Berlanga Silvente, V. (2012). Cómo aplicar las pruebas paramétricas bivariadas $\mathrm{t}$ de Student y ANOVA. Revista d'Innovació i Recerca en Educació, 5(2), 83-100.

Tejada, J. y Peñas, J. (2003). Entrenamiento de base en el fútbol sala. Barcelona: Editorial Paidotribo.

Vasconcelos, A. (1996). Planificación y organización del entrenamiento deportivo. Barcelona: Editorial Paidotribo.

Weineck, J. (2005). Entrenamiento total. Barcelona: Editorial Paidotribo. 